Part of Journal of Research of the National Bureau of Standards, Volume 17, October 1936

\title{
CALCULATIONS OF ELECTRICAL SURGE-GENERATOR CIRCUITS
}

\author{
By Arthur B. Lewis
}

\section{ABSTRACT}

It is pointed out that electrical surge-generator circuits are, essentially, periodically loaded lines. It is then shown that, with the aid of certain reasonable assumptions, the well-known theory dealing with such lines leads directly to expressions for the free periods and damping factors of surge-generator circuits. The resulting equations can be solved exactly in some cases and can be solved approximately in all cases. In certain cases exact expressions can be obtained for the current and voltage amplitudes.

\section{CONTENTS}

II. Surge-voltage generator

1. Statement of the general problem

2. Formal solution of the general problem

3. Illustrative case, exact solution

4. Discussion $\ldots$

III. Surge-current generator

1. Statement of the general problem

2. Formal solution of the general problem

3. Illustrative case, exact solution

4. Illustrative case, approximate solution 601

IV. Conclusion..... 602

\section{INTRODUCTION}

Recent investigations into the behavior of transmission lines and associated equipment, when subjected to voltage and current surges simulating those produced by lightning disturbances, have led to the development and use of the so-called surge-voltage and surge-current generators. A surge-voltage generator consists of a number of capacitors which are charged in parallel and then, by a suitable arrangement of spark-gaps, discharged in series. Figure 1 is a schematic diagram of the circuits of a surge-generator as they function on discharge, the charging circuits being omitted to avoid confusing the figure. If all the capacitors are identical and are initially charged to the same voltage $E_{0}$, the voltage appearing across the terminals of the $n$th mesh will rise to a maximum value of the order of $n E_{0}$ when the gaps are discharged in succession, beginning at the left-hand end. A surge-current generator consists of a number of capacitors which 
are arranged to be charged and discharged in parallel. The current in the terminal mesh of an $n$-section generator will be roughly $n$ times that obtainable from a single capacitor. Figure 3 shows a schematic diagram of a surge-current generator in which, again, the charging circuit is not shown.

The methods of obtaining approximate solutions for such circuits are obvious. If one neglects the internal structure of such a circuit and considers it to consist simply of a lumped effective capacitance, inductance, and resistance, the frequency and amplitude of the fundamental component of current or voltage can be computed with a reasonable degree of accuracy. As an additional approximation one might consider the entire generator to consist of two circuits, a generator with lumped constants coupled to a load circuit. Apparently no analyses of the discharge characteristics of surge-generator circuits have been published which have gone beyond these two obvious approximations, although R. Elsner and C. S. Roys have published complete analyses of the charging characteristics of such circuits. ${ }^{1}$ While serving fairly well to predict the frequency of the fundamental component of current or voltage, this approximation leads to erroneous results when an attempt is made to use it in the computation of critical damping resistances, as will be shown in the section on surge-current generators. In addition, such a simplification entirely neglects the presence of frequencies higher than the fundamental. It is just these higher frequencies, however, which are of particular importance in defining the wave front of such surges.

It is the purpose of this paper to point out that surge-generator circuits are essentially periodically loaded lines, and that the highly developed theory of the periodically loaded line can be applied directly to surge-generator problems. It is shown that straightforward calculations lead to expressions for the free periods and damping factors of surge-generator circuits which can be solved exactly in some cases and approximately in all cases. In certain cases exact expressions can be obtained for the current or voltage amplitudes.

\section{SURGE-VOLTAGE GENERATOR}

\section{STATEMENT OF THE GENERAL PROBLEM}

The problem of the surge-voltage generator can be stated conveniently in terms of the simplified circuit shown in figure 1. A set of $n-1$ identical capacitors of capacitance $C$ are first charged in parallel to a common voltage $E_{0}$. By a suitable arrangement of sphere gaps, as shown, they are then discharged in series through leads having identical resistances $R$ and inductances $L$. The $n$th mesh, of resistance $R_{n}$, inductance $I_{n}$, and capacitance $C_{n}$, contains the equipment under test as well as other measuring apparatus, and is in general different from the other $n-1$ meshes. The capacitance to ground associated with each capacitor and its two spherical terminals is supposed, for simplicity, to be concentrated as shown in figure 1 and to be of common value $C_{b}$. Different locations of this lumped

\footnotetext{
1 P. L. Bellaschi, Trans. Am. Inst. Elec. Engrs. 51, 936-945 (1932); 52, 544-552 (1933); Elec. Eng. 53, 86-94 (1934).

J. C. Dowell and C. M. Foust, Trans. Am. Inst. Elec. Engrs. 52, 537-543 (1933).

B. L. Goodlet, J. Inst. Elec. Eng. (London), 74, 377-396 (1934).

K. B. McEachron and J. L. Thomason, Gen. Elec. Rev. 38, 126-131 (1935).

R. Elsner, Arch. Elektrotech. 29, $655-682$ (1935).
} 
capacitance to ground, as for example, splitting it between the two capacitor terminals, would present no essential mathematical difficulties. We neglect the internal structure of the capacitors, if any, and assume that their internal losses are sufficiently represented by a suitable increase in the lead resistance $R$.

All the capacitors are initially charged to the same voltage, $E_{0}$. If now the first gap is broken down by some artificial means, each of the other gaps in the circuit will break down in its turn as the voltage across it rises to its breakdown value. The problem, then, is to find the charges on the various capacitors as explicit functions of time, subject to the given initial conditions of charge and current. Let $Q_{s}$ represent the charge on the $s$ th main capacitor and $q_{s}$ the charge on the sth capacitor to ground. The method of solution is to set up the equation for the summation of the voltages around each mesh in terms of the charges on the various capacitors. The q's can be eliminated in terms of the $Q$ 's. After certain transformations the resulting $n$ second-order differential equations in the $Q$ 's lead to a trigonometric equation of degree $n$, the secular equation, the roots of which furnish the free periods and damping factors of the system. These roots can be obtained exactly in some cases and can be approximated in every case. The $2 n$ constants necessary to satisfy the original $n$ second-order differential equations determine the amplitudes and phase angles of the charge components. Exact expressions can be found for these constants in certain limiting cases and approximate expressions in certain others.

\section{FORIMAL SOLUTION OF THE GENERAL PROBLEM}

Referring now to figure 1 and writing down the equations for the summation of the voltages around each mesh, the gaps now being considered conducting,

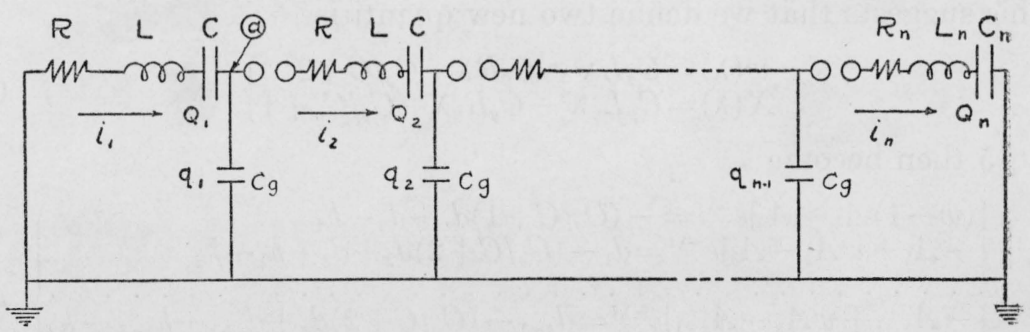

Figdre 1.-Schematic diagram of a surge-voltage generator.

The charging circuits have been omitted to avoid confusing the figure.

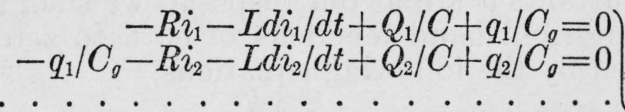

$$
\begin{aligned}
& \left.-q_{s-1} \mid \dot{C}-\dot{R} \dot{i}_{s}-\dot{L} \dot{d} \dot{i}_{s} / d \dot{t}+\dot{Q_{s}} / \dot{C}+\dot{q}_{s} / \dot{C}_{o}=\dot{0}\right\} \\
& \text { - }-\dot{q}_{n-1} / C-\dot{R}_{n} i_{n}-\dot{L}_{n} \dot{d} \dot{i}_{n} / d t+\dot{Q}_{n} / \dot{C}_{n}=\dot{0}
\end{aligned}
$$

Now consider the currents at a representative point, as $a$. By definition

$$
i_{s}=-d Q_{s} / d t
$$


Then at point $a$

Integrating,

$$
-d Q_{s} / d t+d q_{s} / d t=-d Q_{s+1} / d t
$$

At $t=0$,

$$
q_{s}=Q_{s}-Q_{s+1}+h_{s}(\text { constant })
$$

Therefore,

$$
\begin{aligned}
q_{s} & =q_{s, 0} \\
Q_{s} & =Q_{s, 0} \\
Q_{s+1} & =Q_{s+1,0}
\end{aligned}
$$

$$
h_{s}=q_{s, 0}-Q_{s, 0}+Q_{s+1,0}
$$

Substituting eq 2 and 3 in eq 1 we have,

$$
\begin{aligned}
& L d^{2} Q_{1} / d t^{2}+R d Q_{1} / d t+Q_{1} / C+Q_{1} / C_{g}-Q_{2} / C_{g}+h_{1} / C_{g}=0 \\
& -Q_{1} / C_{o}+Q_{2} / C_{o}-h_{1} / C_{g}+L d^{2} Q_{2} / d t^{2}+R d Q_{2} / d t+Q_{2} / C \\
& +Q_{2} / C_{g}-Q_{3} / C_{g}+h_{2} / C_{g}=0
\end{aligned}
$$

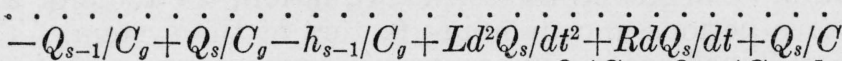

$$
\begin{aligned}
& +Q_{s} / C_{o}-Q_{s+1} / C_{o}+h_{s} / C_{o}=0
\end{aligned}
$$

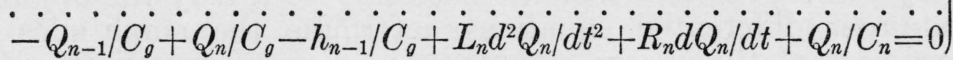

Now make the usual exponential substitution

$$
Q_{s}=d_{s}+A_{s} e^{-\lambda t}
$$

where $d_{s}, A_{s}$, and $\lambda$ are constants, as yet undetermined. ${ }^{2}$ Applying eq 6 to the typical equation of eq 5 we obtain,

$$
\begin{aligned}
& -h_{s-1}-d_{s-1}+\left[-A_{s-1}+\left(C_{g} L \lambda^{2}-C_{g} R \lambda+C_{g} / C+2\right) A_{s}-A_{s+1}\right] e^{-\lambda t} \\
& +\left(C_{g} / C+2\right) d_{s}-d_{s+1}+h_{s}=0
\end{aligned}
$$

This suggests that we define two new quantities

$$
\left.\begin{array}{l}
w(\lambda)=C_{0} L \lambda^{2}-C_{g} R \lambda+C_{o} / C+2 \\
X(\lambda)=C_{0} L_{n} \lambda^{2}-C_{0} R_{n} \lambda+C_{o} / C_{n}+1
\end{array}\right\}
$$

Eq 5 then become

$$
\begin{aligned}
& {\left[(w-1) A_{1}-A_{2}\right] e^{-\lambda t}=-\left(C_{g} / C+1\right) d_{1}+d_{2}-h_{1}} \\
& {\left[-A_{1}+w A_{2}-A_{3}\right] e^{-\lambda t}=d_{1}-\left(C_{g} / C+2\right) d_{2}+d_{3}+h_{1}-h_{2}} \\
& {\left[\dot{-} \dot{A}_{s-1}+\dot{w A_{s}}-\dot{A}_{s+1}\right] e^{-\lambda i}=\dot{d_{s-1}}-\dot{\left(C_{o} / C+2\right)} \dot{d_{s}}+\dot{d}_{s+1}+\dot{h}_{s-1}-\hat{h}_{s}} \\
& {\left[-\dot{A}_{n-1} \dot{+} \dot{X} \dot{A_{n}}\right] e^{-\lambda t}=\dot{d_{n-1}} \dot{-}(\dot{C} g / \dot{C} \dot{+1}) \dot{d_{n}} \dot{+} \dot{h_{n-1}}}
\end{aligned}
$$

Since the constants $d$ are at our disposal, we shall so choose them as to reduce the right-hand members of eq 8 to zero. The $d$ 's are therefore defined by the following equations,

2 Strictly speaking there will be $2 n \lambda^{\prime} s$ and $Q_{s}$ will be of the form $Q_{s}=\sum_{k=1}^{2 n} A_{s k} e^{-\lambda_{k} t}$. For the time being we shall omit the subscript $k$. Strickly speaking also $A_{s}$ is not necessarily a constant. In case a given $\lambda_{r}$ is a double root of the secular equation, that is, if the $r$ th frequency component is critically damped, the corresponding $A_{s r}$ is a linear function of time of the form $(A t+B)$. See, for example, Ames and Murnaghan, Theoretical Mechanics, page 124 (Ginn and Co., 1929). 


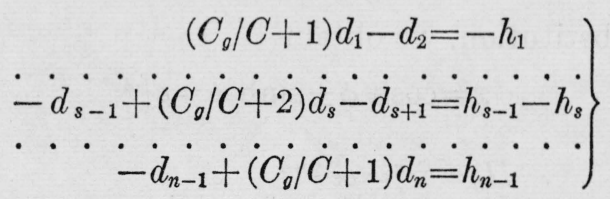

With the $d$ 's having the values determined by eq 9 the solution of eq 8 reduces to the solution of the following equations:

$$
\begin{aligned}
& \begin{array}{r}
(w-1) A_{1}-A_{2}=0 \\
-A_{1}+w A_{2}-A_{3}=0
\end{array} \\
& \because \ddot{A_{s-1}}+\ddot{w} \dot{A}_{s}-\ddot{A}_{s+1}=0 \\
& \because \ddot{A_{n-1}} \ddot{+} \ddot{X} \ddot{A_{n}}=0
\end{aligned}
$$

The condition that a solution other than zero shall exist for the $A$ 's requires, as usual, that the determinant of the coefficients shall vanish. Let us call this determinant $D_{n}$. The condition for a solution then reduces to

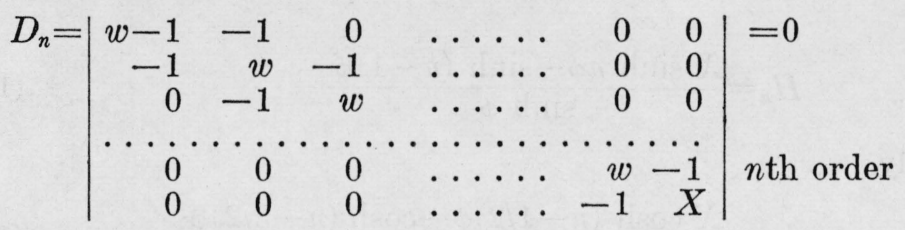

Let us also define

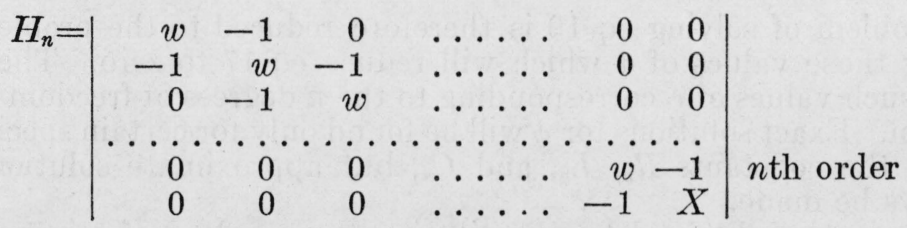

Expanding $D_{n}$ on its first column, we obtain

$$
D_{n}=(w-1) H_{n-1}-H_{n-2}
$$

Expanding $H_{n}$ similarly, we obtain

$$
H_{n}=w H_{n-1}-H_{n-2}
$$

The method of handling such difference equations and the application of the results to electrical problems has been discussed by Wheeler and Murnaghan. ${ }^{3}$ Let us make the trial substitution $H_{n}=c x^{n}$, where $c$ is any constant. Substituting in eq 14 we obtain

whence

$$
\begin{aligned}
x^{2}-w x & =-1 \\
x & =w / 2 \pm\left[w^{2} / 4-1\right]^{1 / 2}
\end{aligned}
$$

This suggests the substitution

$$
w / 2=\cosh \phi
$$

\footnotetext{
${ }^{3}$ H. A. Wheeler and F. D. Murnaghan, Phil. Mag. [7] 6, 146-174 (July 1928).
} 
Making this substitution, we obtain

Hence

$$
x=\cosh \phi \pm \sinh \phi=e^{ \pm \phi}
$$

or

$$
\begin{aligned}
& H_{n}=c x^{n}=c e^{ \pm n \phi} \\
& H_{n}=c_{1} \cosh n \phi+c_{2} \sinh n \phi
\end{aligned}
$$

The values of $c_{1}$ and $c_{2}$ may be determined from the values assumed by $H_{n}$ when $n=0$ and $n=1$. From eq 12 and 14 we have $H_{2}=w X-1=$ $w H_{1}-H_{0}$. Therefore

$$
\begin{aligned}
& H_{0}=1 \\
& H_{1}=X
\end{aligned}
$$

Using these values we readily find

$$
\begin{aligned}
& c_{1}=1 \\
& c_{2}=\frac{X-\cosh \phi}{\sinh \phi}
\end{aligned}
$$

so that

$$
H_{n}=\frac{X \sinh n \phi-\sinh (n-1) \phi}{\sinh \phi}
$$

And finally

$$
D_{n}=\frac{X \cosh (n-1 / 2) \phi-\cosh (n-3 / 2) \phi}{\cosh \phi / 2}
$$

The problem of solving eq 10 is therefore reduced to the problem of finding those values of $\phi$ which will reduce eq 17 to zero. There will be $n$ such values of $\phi$ corresponding to the $n$ degrees of freedom of the system. Exact solutions for $\phi$ will be found only for certain special values of the constants $R_{n}, L_{n}$, and $C_{n}$, but approximate solutions can always be made.

Values of $\lambda$ are obtained by substituting these values of $\phi$ in eq 7 and (15), from which we obtain

$$
\lambda_{k, 1}, \lambda_{k, 2}=R / 2 L \pm\left[R^{2} / 4 L^{2}-1 / C L+\left(4 / C_{0} L\right) \sinh ^{2} \phi_{k} / 2\right]^{\frac{1}{2}}
$$

there being $n$ values of $k$ corresponding to the $n$ possible values of $\phi$.

It can now be verified by direct substitution that eq 10 will be satisfied for all possible values of $\phi$ if the $A$ 's are of the form

$$
A_{s}=P_{k} \cosh (s-1 / 2) \phi_{k}
$$

where the $P_{k}$ are constants.

Therefore, the final solution of eq 5 is of the form

$$
Q_{s}=d_{s}+\sum_{k}\left[P_{k, 1} e^{-\lambda_{k, 1} t}+P_{k, 2} e^{-\lambda_{k, 2} t}\right] \cosh (s-1 / 2) \phi
$$

The constant $d_{s}$ is evaluated from eq 9 and the constants $P_{k, 1}$ and $P_{k, 2}$ are determined by the initial conditions, that is, by the values of $Q_{s}$ and $i_{s}$ at $t=0$. With the evaluation of these constants the problem is completely solved, for with the aid of eq 20 we can now compute the voltage drop across any element of the circuit of figure 1 as an explicit function of time. 


\section{ILLUSTRATIVE CASE, EXACT SOLUTION}

The application of the preceding formula is best illustrated by means of a specific example. For simplicity, a case has been chosen in which an exact solution is possible. Other special cases leading to exact solutions can be discovered with little difficulty. These exact solutions may be found to be of little practical importance since the constants of the terminal mesh, including as they do the constants of the apparatus under test, cannot in general be chosen arbitrarily.

Referring to figure 1, let us assume that

$$
R_{n}=R, L_{n}=L, C_{n}=\frac{C C_{g}}{C+C_{g}}
$$

This is assuming that the terminal section is identical with the typical section, the capacitance $C_{n}$ being replaced by a capacitance $\dot{C}$ in series with a capacitance $C_{g}$. Substituting these values in eq 7 we find

$$
X=w=2 \cosh \phi
$$

Substituting this value in eq 17 , we find

$$
D_{n}=\frac{\cosh (n+1 / 2) \phi}{\cosh \phi / 2}
$$

The condition for a solution is that $D_{n}=0$, or

Hence

$$
\frac{\cosh (n+1 / 2) \phi}{\cosh \phi / 2}=0
$$

or, if $i \theta=\phi \quad \theta_{k}=\frac{2 k+1}{2 n+1} \pi, k=0,1,2,3, \ldots . n-1$

Referring to eq 18 , we have

$$
\lambda_{k, 1}, \lambda_{k, 2}=R / 2 L \pm\left[R^{2} / 4 L^{2}-1 / C L-\left(4 / C_{\theta} L\right) \sin ^{2} \theta_{k} / 2\right]^{1 / 2}
$$

Three cases are to be distinguished, as usual, depending upon whether the radical of eq 22 is real, zero, or imaginary. In the first case ( $R$ greater than necessary for critical damping) the amplitude substitutions will be as given in eq 20 . In the second case ( $R$ equal to the critical damping resistance for a given frequency) the substitution will be of the form (See footnote 2, page 588.)

$$
\left(P_{r, 1} t+P_{r, 2}\right) e^{-\lambda_{r} t} \cos (s-1 / 2) \theta_{r} .
$$

In the third case ( $R$ less than that necessary for critical damping) the $\lambda$ 's will be of the form

where

$$
\lambda_{k}=\mu \pm 2 \pi i \nu_{k}
$$

$$
\begin{aligned}
\mu & =R / 2 L \\
2 \pi \nu_{k} & =\left[-R^{2} / 4 L^{2}+1 / C L+\left(4 / C_{\theta} L\right) \sin { }^{2} \theta_{k} / 2\right]^{1 / 2}
\end{aligned}
$$

In this case the substitution corresponding to eq 20 will be of the form

$$
P_{k} \cos (s-1 / 2) \theta e^{-\mu t} \cos \left(2 \pi \nu_{k} t-\alpha_{k}\right),
$$

where $P_{k}$ and $\alpha_{k}$ are now the unknown constants. 
Suppose now that the value of $R$ is such as to critically damp the $r$ th frequency component. We can then write a general expression for the charge on the sth capacitor in the following form:

$$
\left.\begin{array}{rl}
Q_{s}=d_{s} & +\sum_{k=0}^{r-1}\left(P_{k, 1} e^{-\lambda_{k, 1} t}+P_{k, 2} e^{-\lambda_{k, 2} t}\right) \cos (s-1 / 2) \theta_{k} \\
& +\left(P_{r, 1} t+P_{r, 2}\right) e^{-\lambda_{r} t} \cos (s-1 / 2) \theta_{r} \\
& +\sum_{k=r+1}^{n-1} P_{k} \cos (s-1 / 2) \theta_{k} e^{-\mu t} \cos \left(2 \pi \nu_{k} t-\alpha_{k}\right)
\end{array}\right\}
$$

The $2 n$ constants are determined from the initial conditions, that is at $t=0$,

$$
\begin{aligned}
Q_{s} & =Q_{s, 0} \\
i_{s} & =i_{s, 0}
\end{aligned}
$$

The problem of determining these $2 n$ constants is the standard problem of passing an $n$ term trigonometric sum through $n$ arbitrarily chosen points. If the $n$ points are equally spaced (that is, if the solution of eq 17 yields exact values of $\theta$ of the form $\theta=\frac{k \pi}{n}, \frac{2 k+1}{2 n+1} \pi$, etc.) the solutions can be written down immediately. ${ }^{4}$ The condition that $Q_{s}=Q_{s, 0}$ at $t=0$ leads to the solutions of the following identical form for $\left(P_{\mathrm{k}, 1}+P_{k, 2}\right), P_{r, 2}$, and $P_{k} \cos \alpha_{k}$ (for the case where $\left.\theta_{k}=\frac{2 k+1}{2 n+1} \pi\right)$

$$
P_{k} \cos \alpha_{k}=4 /(2 n+1) \sum_{s=1}^{n}\left(Q_{s, 0}-d_{s}\right) \cos (s-1 / 2) \theta_{k} .
$$

The second initial condition, $i_{s}=i_{s, 0}$ at $t=0$, leads to similar solutions for the same pairs of constants, from which the individual constants can be evaluated. If the initial conditions are known the summations of eq 25 can always be carried through and the constants determined.

The exact determination of these initial conditions is a matter of some difficulty in the case of a surge-voltage generator. It is not correct to assume that at time zero all charges are equal and all currents zero, although this represents the limiting case of all gaps closing simultaneously. Actually, the breakdown of the gaps is successive, and the initial time for the whole system should be taken as the moment of breakdown of the last gap. At this moment the charges on the preceding capacitors are not identical and the currents in the preceding meshes are not zero. To compute the initial conditions exactly the following procedure must be followed. First, compute the voltage above ground of the terminal of the second section of the generator (that is, point $a$, fig. 1) after the first gap has been closed and before the second gap has broken down. This computation can be carried out exactly since there is only one gap in this circuit and it is arbitrarily closed at time zero, at which time both initial charges are equal and both initial currents are zero. Now, having a knowledge of the voltage at which the second gap will break down, or having made a reasonable assumption as to that voltage, we compute the charges and currents existing at the moment that break-

4 W. E. Byerly, Fourier's Series and Spherical Harmonics, chapter II. (Ginn and Co., 1895.) 
down voltage is reached at the second gap. This moment is taken as the new origin of time measurement in dealing with the threesection network now in the circuit, and these charges and currents are the required initial conditions. We repeat the above process for the third gap and for each of the other gaps in turn until finally we are able to compute all the charges and all the currents at the moment of breakdown of the last gap. It is readily seen that the rigorous computation of the voltage across the terminals of an $n$-section generator becomes very laborious if $n$ is very large, say 20 or 30 .

To make these ideas somewhat more specific, sample calculations have been carried out on a three-section network having the following circuit constants:

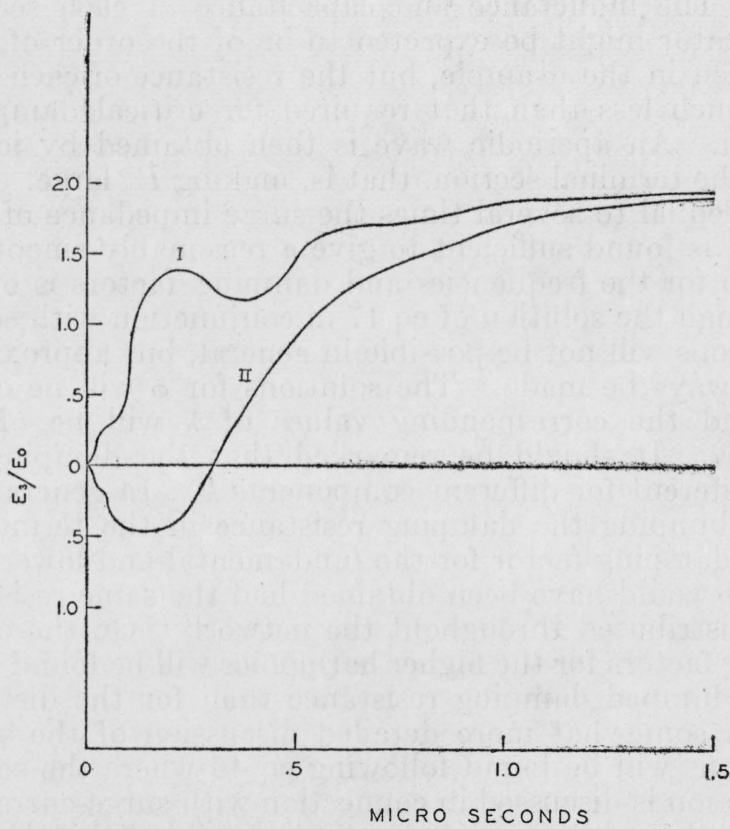

Figure 2.-Voltage surge of a two-section generator discharging into a previously uncharged terminal section.

Curve $I$, both gaps closed simultaneously.

Curve $I$, second gap closed $1 \times 10^{-7}$ seconds after first gap.

$$
\begin{aligned}
L=L_{n} & =5 \times 10^{-6} \mathrm{~h} . \\
R=R_{n} & =40.5 \text { ohms. } \\
C & =0.25 \times 10^{-6} \text { farads. } \\
C_{g} & =0.25 \times 10^{-8} \text { farads. } \\
C_{n} & =\frac{C C_{g}}{C+C_{g}} \\
E_{0} & =\text { initial charging voltage. }
\end{aligned}
$$

The value of resistance used is just sufficient to critically damp the fundamental of the three-section network. We have here a twosection generator discharging into a previously uncharged terminal circuit. 
In figure 2 are plotted curves of $E_{3} / E_{0}$ against time, $E_{3}$ being the voltage across the capacitor $C_{g}$ in the terminal circuit. Curve $I$ is plotted for the limiting case in which both gaps are closed simultaneously. Curve $I I$ is plotted for the case in which the second gap closes 0.1 microsecond after the first gap. In this time, the voltage across the second gap will have risen to about $1.25 E_{0}$. The difference between these curves indicates the importance of initial conditions, that is, gap settings, on the shape of the voltage wave front.

\section{DISCUSSION}

In surge-voltage generators as used in practical work the situation is somewhat more complicated than is indicated in the simple example just cited. The inductance and capacitance of each section of an actual generator might be expected to be of the order of magnitude of those given in the example, but the resistance of each section, $R$, might be much less than that required for critical damping of the fundamental. An aperiodic wave is then obtained by inserting resistance in the terminal section, that is, making $R_{n}$ large. Ordinarily a resistance equal to several times the surge impedance of the circuit $\left(L_{\text {total }} / C_{1}\right)^{1 / 2}$, is found sufficient to give a reasonably smooth wave. ${ }^{5}$

$\mathrm{A}$ solution for the frequencies and damping factors is obtained, as before, through the solution of eq 17 in conjunction with eq 7 and 15 . Exact solutions will not be possible in general, but approximate solutions can always be made. The solutions for $\phi$ will be of the form $\phi=x \pm i y$ and the corresponding values of $\lambda$ will be of the form $\lambda_{k}=\mu_{k} \pm 2 \pi i \nu_{k}$. It should be remarked that the damping constant $\mu_{k}$ is now different for different components $k$. In general, it will be found that lumping the damping resistance in the terminal section results in a damping factor for the fundamental and lower harmonics greater than would have been obtained had the same resistance been uniformly distributed throughout the network. On the other hand, the damping factors for the higher harmonics will be found to be much less for the lumped damping resistance than for the distributed resistance. A somewhat more detailed discussion of the solutions of such equations will be found following eq 46 where the solution of a similar equation is discussed in connection with surge-current circuits. As is shown there, the circuit constants in any particular case may suggest valid simplifications and approximations which greatly facilitate the computations.

Exact solutions for the amplitude factors $P_{k}$, see eq 20, cannot be obtained in general. With a knowledge of the proper form of solution, however, and the correct values of the $\lambda$ 's, it will be possible to compute, by the ordinary methods of wave analysis, the amplitude factors $P_{k}$ necessary to fit a given surge-voltage oscillogram. Even this meager information may prove valuable, however. It will enable one, from data obtained on the well-defined crest and tail of a voltage wave, to reproduce the sharply rising front of the voltage wave which is actually of much importance but is often poorly defined on the oscillograms.

\footnotetext{
B P. L. Bellaschi, Trans. Am. Inst. Elec. Engrs. 51, 936-945 (1932).
} 


\section{SURGE-CURRENT GENERATOR}

\section{STATEIMENT OF THE GENERAL PROBLEM}

The problem to be solved in connection with some types of the surge-current generator can be stated conveniently in terms of the simplified circuit shown in figure 3 . The $n$ identical capacitors com-

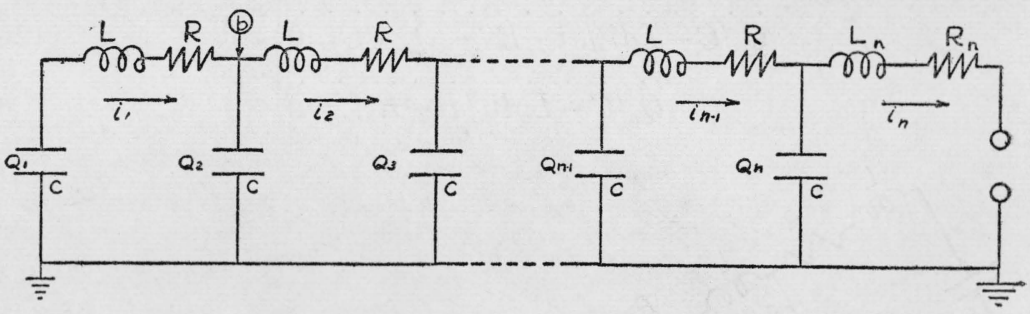

Figure 3.-Schematic diagram of a surge-current generator.

The charging circuits have been omitted to avoid confusing the figure.

prising this generator are connected in parallel by identical leads having resistances $R$ and inductances $L$. The $n$ th, or terminal, mesh, which in practice contains the apparatus under test as well as other measuring equipment, is closed through a resistance $R_{n}$ and an inductance $L_{n}$ which is, in general, different from the resistance and inductance $(R, L)$ of the other $n-1$ meshes. As actually used, a surge-current generator will consist of two or more units, such as shown in figure 3 , connected in parallel to a common sphere gap and load, as are shown in figure $4 .^{6}$ If the parallel circuits of figure 4 are identical, and if we can neglect the effect of mutual inductance between leads, it is obvious that the currents in the parallel circuits will be identical at all times and that the current through the load resistance will be simply three times that flowing in any one of the parallel circuits. The solution for the case of a symmetrical circuit, such as figure 4, therefore reduces essentially to the solution of the circuit of figure 3 . As before, we neglect the internal structure, if any, of the capacitors and represent their internal losses by a suitable series resistance, and neglect the change of resistance of the leads with frequency. If identical charges $Q_{0}$ are placed on each of the capacitors and the $n$th mesh is then closed through its inductance and resistance, it is required to find the charges on the various capacitors and the currents in the various meshes as explicit functions of time. We are particularly interested here in the current in the $n$th mesh. The method of solution will closely parallel that used in connection with the surge-voltage generator in section II.

6 P. L. Bellaschi, Elec. Eng. 53, 86 (1934). K. B. McEachron and J. L. Thomason, Gen. Elec. Rev. 38, 126 (1935). 


\section{FORMAL SOLUTION OF THE GENERAL PROBLEM}

Referring now to figure 3 and writing down the equations for the summation of the voltages around each mesh we have, if $Q_{s}$ is the charge on the sth capacitor and $i_{s}$ the corresponding current:

$$
\begin{aligned}
& Q_{1} / C-L d i_{1} / d t-R i_{1}-Q_{2} / C=0 \\
& \dot{Q}_{s} / \dot{C}-\dot{L} \dot{d i}_{s} \dot{d t}-\dot{R} i_{s}-\dot{Q}_{s+1} \dot{C}=0 \\
& \text { - } \dot{Q_{n}} / \dot{C}-\dot{L_{n}} d \dot{i_{n}} / \dot{d} t \dot{-R_{n}} \dot{i_{n}}=\dot{0}
\end{aligned}
$$

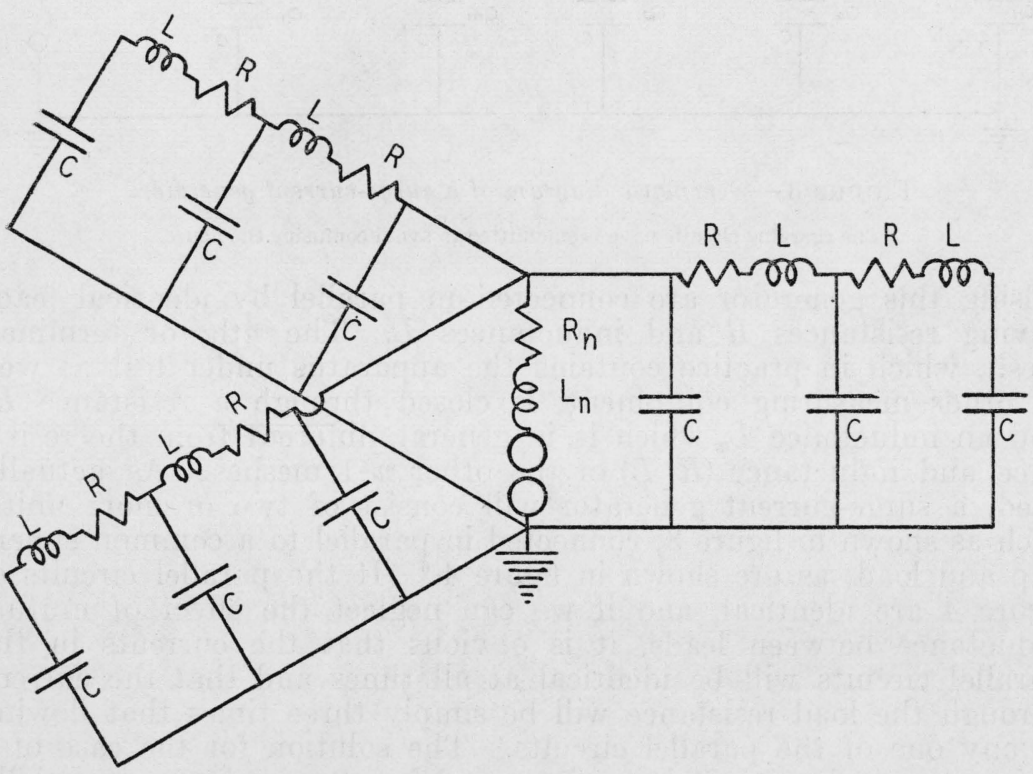

FiguRE 4.-Schematic diagram of a surge-current generator using three identical circuits in parallel.

Now, noting the currents at point $b$, we can write

$$
\begin{aligned}
i_{1} & =-d Q_{1} / d t \\
i_{1}-i_{2}-d Q_{2} / d t & =0
\end{aligned}
$$

or

$$
i_{2}=-\left(d Q_{1} / d t+d Q_{2} / d t\right)
$$

and, in general,

$$
i_{s}=-\sum_{r=1}^{s} d Q_{\tau} / d t .
$$


Let us now make the usual exponential substitution

$$
Q_{s}=B_{s} e^{-\lambda t}
$$

where $B_{s}$ and $\lambda$ are undetermined constants (see footnote 2, p. 588).

Substituting eq 27 and 28 in the typical equation of eq 26 and making a few obvious rearrangements, we have

$$
\left(\lambda^{2} L C-\lambda R C\right)_{\tau=1}^{s-1} \sum_{r}+\left(\lambda^{2} L C-\lambda R C+1\right) B_{s}-B_{s+1}=0
$$

This suggests the following definitions:

$$
\left.\begin{array}{l}
u=\lambda^{2} L C-\lambda R C+1 \\
Y=\lambda^{2} L_{n} C-\lambda R_{n} C+1
\end{array}\right\}
$$

Eq 26 can then be put in the form:

$$
\left.\begin{array}{r}
u B_{1}-B_{2}=0 \\
(u-1)_{r=1}^{s-1} \sum_{r}+u B_{s}-B_{s+1}=0 \\
(Y-1)_{r=1}^{n-1} \sum B_{r}+Y B_{n}=0
\end{array}\right\}
$$

The condition for a solution giving values for the $B$ 's other than zero is, as usual, that the determinant of the coefficients shall vanish. This $n$ th-order determinant, which leads to the secular equation, we shall call $F_{n}$. The determinantal equation is:

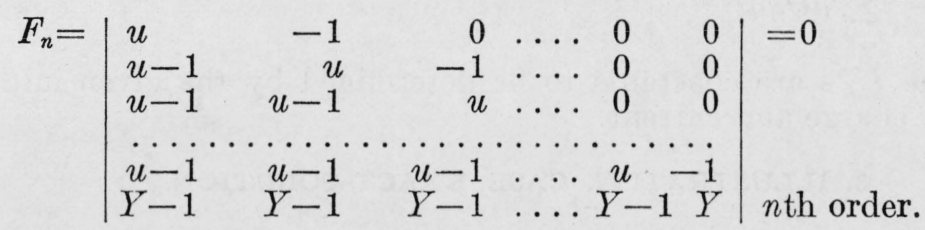

Let us also define the following determinant which is obtained on expanding $F_{n}$ :

$$
K_{n}=\left|\begin{array}{ccrccr}
u-1 & -1 & 0 & \ldots & 0 & 0 \\
u-1 & u & -1 & \ldots & 0 & 0 \\
u-1 & u-1 & u & \ldots & 0 & 0 \\
\ldots \ldots & \ldots & \ldots & \ldots & \ldots & \ldots \\
u-1 & u-1 & u-1 & \ldots & u & -1 \\
Y-1 & Y-1 & Y-1 & \ldots & Y-1 & Y
\end{array}\right| \text { nth order. }
$$

Expanding eq 31 on the first row, we obtain

$$
F_{n}=u F_{n-1}+K_{n-1}
$$

Expanding eq 32 on the first row, we obtain

whence

$$
\begin{aligned}
K_{n} & =(u-1) F_{n-1}+K_{n-1} \\
K_{n-1} & =-F_{n-2}+\left(u F_{n-2}+K_{n-2}\right)
\end{aligned}
$$$$
=F_{n-1}-F_{n-2} \text {. }
$$

Therefore,

$$
F_{n}=(u+1) F_{n-1}-F_{n-2} \text {. }
$$


$\mathrm{Eq}$ "33 is of exactly the same form as eq 14 and the method of solution of the two equations is identical. If we make the definition

$$
(u+1) / 2=\cosh \xi
$$

we find that eq 33 can be put in the form

$$
F_{n}=\frac{Y \sinh n \xi-\sinh (n-1) \xi}{\sinh \xi}
$$

The problem of solving eq 26 is now reduced to the problem of finding those values of $\xi$ which will reduce the right-hand member of eq 35 to zero. There will be $n$ such values of $\xi$ corresponding to the $n$ degrees of freedom of the system.

Values of $\lambda$ are obtained by substituting these values of $\xi$ in eq 29 and 34 from which we obtain

$$
\lambda_{k, 1}, \lambda_{k, 2}=R / 2 L \pm\left[R^{2} / 4 L^{2}+(4 / L C) \sinh ^{2} \frac{\xi_{k}}{2}\right]^{\frac{1}{1}}
$$

there being $n$ values of $k$ corresponding to the $n$ possible values of $\xi$.

It can now be verified by direct substitution that eq 30 are satisfied for all possible values of $\xi$ if the $B$ 's are of the form

$$
B_{s}=U_{k} \cosh (s-1 / 2) \xi_{k} \text {. }
$$

The final solution of our problem is therefore given by

$$
Q_{s}=\sum_{k}\left[U_{k, 1} e^{-\lambda_{k, 1} t}+U_{k, 2} e^{-\lambda_{k, 2} t}\right] \cosh (s-1 / 2) \xi_{k}
$$

and $i_{s}=-\sum_{r=1}^{s} d Q_{r} / d t$

where the $U_{k}$ 's are constants to be determined by the given initial values of charge and current.

\section{ILLUSTRATIVE CASE, EXACT SOLUTION}

The application of the preceding formulas is best illustrated by means of a specific example. For simplicity, a case has been chosen in which an exact solution is possible. Other special cases leading to exact solutions can be discovered with little difficulty.

Referring to figure 3, let us assume that

$$
R_{n}=R \text { and } L_{n}=L .
$$

This is assuming that the terminal section is identical with the typical section. Substituting these values in eq 29 and 35, we obtain

$$
Y=u=2 \cosh \xi-1
$$

and

$$
F_{n}=\frac{\cosh (n+1 / 2) \xi}{\cosh \xi / 2}
$$

and the condition for a solution, $F_{n}=0$, reduces to

$$
\frac{\cosh (n+1 / 2) \xi}{\cosh \xi / 2}=0
$$


Hence

$$
\xi_{k}=\frac{2 k+1}{2 n+1} \pi i
$$

or, if $\xi=i \psi$

$$
\psi_{k}=\frac{2 k+1}{2 n+1} \pi
$$

$$
k=0,1,2, \ldots n-1
$$

Referring to eq 36 we have

$$
\lambda_{k, 1}, \lambda_{k, 2}=R / 2 L \pm\left[R^{2} / 4 L^{2}-(4 / L C) \sin ^{2} \frac{\psi_{k}}{2}\right]^{1 / 2} .
$$

As before, we distinguish three cases depending upon whether $R$ is greater than, equal to, or less than the value necessary to critically damp a given frequency. For values of $R$ less than the critical damping value, we will have

$$
\lambda_{k, 1}, \lambda_{k, 2}=\mu \pm 2 \pi i \nu_{k}
$$

where

$$
\mu=R / 2 L
$$

$$
2 \pi \nu_{k}=\left[-R^{2} / 4 L^{2}+(4 / L C) \sin ^{2} \frac{\psi_{k}}{2}\right]^{1 / 2} .
$$

If now the value of $R$ is such as to critically damp the $r$ th frequency component, we can rewrite eq 38 in a form similar to eq 24 .

$$
\begin{aligned}
Q_{s}=\sum_{k=0}^{r-1}[ & {\left[U_{k, 1} e^{-\lambda_{k, 1} t}+U_{k, 2} e^{-\lambda_{k, 2} t}\right] \cos (s-1 / 2) \psi_{k} } \\
& +\left[U_{r, 1} t+U_{r, 2}\right] e^{-\lambda_{r} t} \cos (s-1 / 2) \psi_{r} \\
& +\sum_{k=r+1}^{n-1} U_{k} \cos (s-1 / 2) \psi_{k} e^{-\mu t} \cos \left(2 \pi \nu_{k} t-\beta_{k}\right) .
\end{aligned}
$$

The constants $U$ and $\beta$ are determined, as before, by the initial conditions, which are, in this case at $t=0$

$$
\begin{gathered}
Q_{s}=Q_{0} \\
i_{s}=0
\end{gathered}
$$

If $R$ is less than the critical damping resistance for the fundamental, these conditions lead to the following values for the constants,

$$
\begin{aligned}
& \beta_{k}=\tan ^{-1} \mu / 2 \pi \nu_{k} \\
& U_{k}=\left[(-1)^{k} 2 Q_{0} /(2 n+1) \cos \beta_{k}\right] \cot \frac{\psi_{k}}{2}
\end{aligned}
$$

whence

$Q_{s}=\sum_{k=0}^{n-1}(-1)^{k}\left[2 Q_{0} /(2 n+1) \cos \beta_{k}\right] \cot \frac{\psi_{k}}{2} \cos (s-1 / 2) \psi_{k} e^{-\mu t} \cos \left(2 \pi \nu_{k} t-\beta_{k}\right)$ and finally

$$
i_{n}=2 Q_{0} /(2 n+1)(L C)^{1 / 2} \sum_{k=0}^{n-1} \sin \frac{\psi_{k}}{2} / \cos \beta_{k} \cdot \cot ^{2} \frac{\psi_{k}}{2} e^{-\mu t} \sin 2 \pi \nu_{k} t .
$$


Let us assume, as a practical example, a generator having the following constants,

$$
\begin{aligned}
& n=10, L=1 \times 10^{-6} h \\
& C=2.4 \times 10^{-7} f, R=0.01 \mathrm{ohm} .
\end{aligned}
$$

These constants approximate those that might be used in an actual generator. Using these data we find

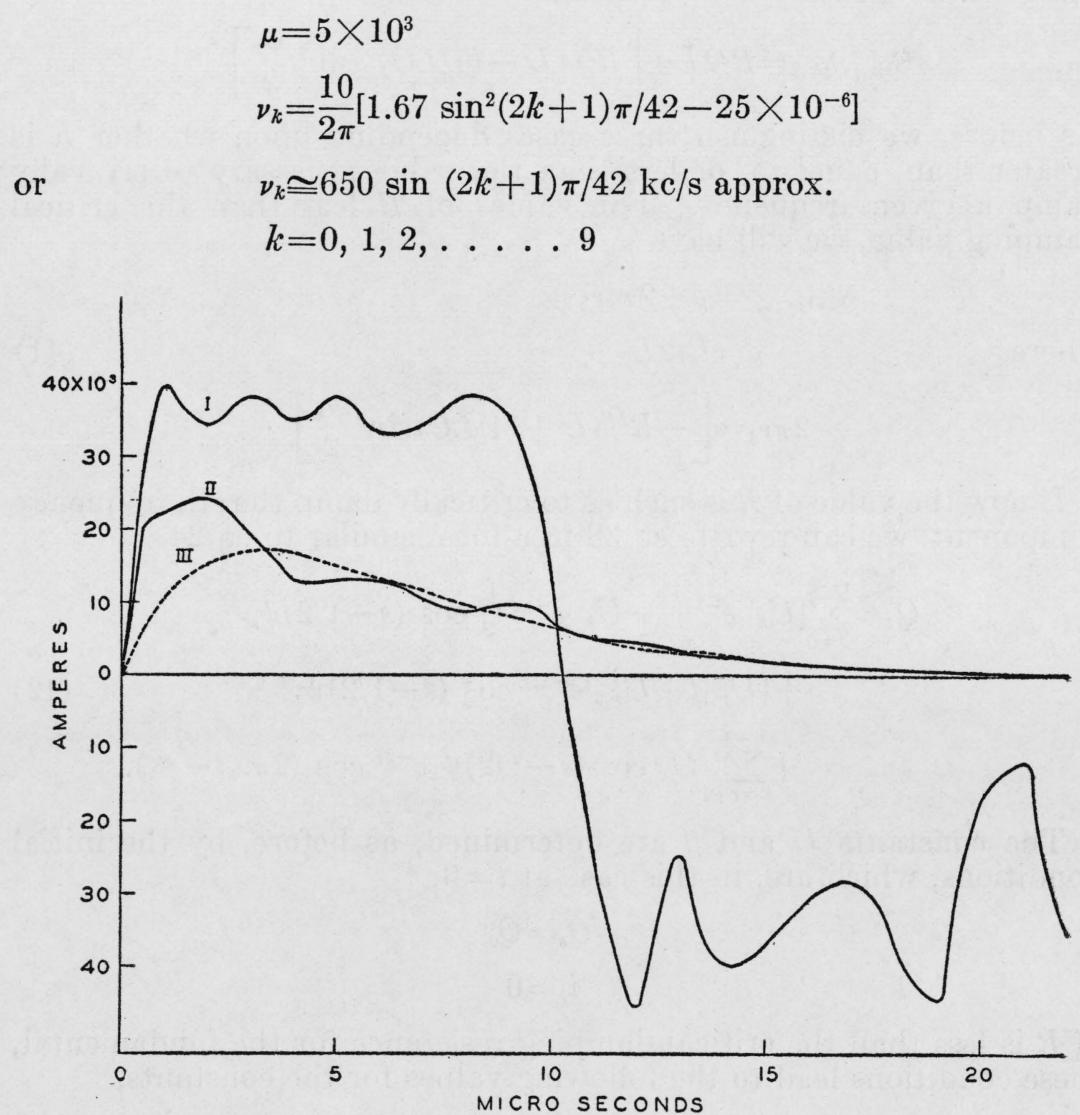

FiguRe 5.-Current surge from a 10-section surge-current generator.

Curve $I$, distributed resistance of $0.01 \mathrm{ohm}$ per section, fundamental very much underdamped. Curve $I I$, distributed resistance of $0.61 \mathrm{ohm}$ per section, fundamental critically damped. Curve III, the critically-damped fundamental corresponding to curve $I I$.

This gives a fundamental, $\nu_{0}$, of $48.5 \mathrm{kc} / \mathrm{s}$, which is to be compared with $32.4 \mathrm{kc} / \mathrm{s}$, which would be computed by considering all the constants of the generator lumped and computing the resulting frequency.

In figure 5 , curve $I, i_{n}$, the current in the terminal mesh, is plotted against time, using the circuit constants just given. In figure 5, curve $I I, i_{n}$ has been plotted for a generator having the same constants, except that $R=0.61 \mathrm{ohm}$. This value of resistance is just sufficient to critically damp the fundamental. In this case $\mu$ (critical) $=30.4 \times 10^{4}$. Curve $I I I$ represents the critically-damped fundamental for this case. 


\section{ILLUSTRATIVE CASE, APPROXIMATE SOLUTION}

In actual practice it is customary to make the internal resistance of the generator $(R)$ as small as possible and to achieve the necessary damping by making the terminal resistance $\left(R_{n}\right)$ sufficiently large. These conditions allow certain simplifying assumptions to be made in the general solution of eq 35 . It is well known, and can be verified in the numerical example of the last section, that if the damping factor is small, the damping affects the frequency as a second-order correction only. Neglecting the internal resistance of the generator for the moment, we have, from eq 29 and 34 ,

$$
\lambda= \pm 2 /(L C)^{1 / 2} \cdot \sinh \xi / 2
$$

and eq 35 then becomes

$$
\frac{\cosh (n-1 / 2) \xi+\left[2 L_{n} / L \cdot \sinh \xi / 2 \mp R_{n}(C / L)^{1 / 2}\right] \sinh n \xi}{\cosh \xi / 2}=0
$$

This equation can be solved approximately by any one of the standard methods. Its roots will be of the form

$$
\xi_{k}=x_{k} \pm i y_{k}
$$

and the $\lambda$ 's will be of the form

$$
\lambda_{k, 1}, \lambda_{k, 2}= \pm 2 /(L C)^{1 / 2} \cdot \sinh \frac{\xi_{k}}{2-}=\mu_{k} \pm 2 \pi i \nu_{k},
$$

and we have, in addition, the small damping arising from the internal resistance $\mu_{o}=\mathrm{R} / 2 \mathrm{~L}$.

If we make the additional assumption that $L_{n}=L$, that is, that we add pure resistance only to the terminal section, eq 46 simplifies to

$$
\frac{\cosh (n+1 / 2) \xi \mp R_{n}(C / L)^{1 / 2} \sinh n \xi}{\cosh \xi / 2}=0
$$

Approximate solutions can be written down for two limiting cases

$$
\begin{aligned}
& R_{n} \text { very small } \xi_{k}=\frac{2 k+1}{2 n+1} \pi i k=0,1,2, \ldots n-1 \\
& R_{n} \text { very large } \xi_{k}=\frac{k}{n} \pi i \quad k=1,2, \ldots n-1
\end{aligned}
$$

Taking one of these approximations as a value of $i y$ above, we can enter eq 47 and find approximate values for $x_{k}$. Using these values of $x_{k}$, we can now compute a second approximation to the true values of $y_{k}$. This process may be repeated if necessary. The value of $y$ corresponding to $k=0$ may sometimes have to be obtained by a separate evaluation. If we wish to find the value of $R_{n}$ necessary to critically damp the fundamental, we solve equation 47 graphically under the assumption that $y_{0}$ is a small quantity the squares of which can be neglected and find that value of $R_{1}$ which just causes $y_{0}$ to vanish.

$89799-36-8$ 
Sample calculations have been carried out on the 10 -section generator of the last section under the conditions $L_{n}=L, R=0.01$ ohms, $R_{n}=2.3 \mathrm{obms}$ (critical damping for the fundamental). In figure 6 are plotted, as solid lines, the damping factors, $\mu_{k}$, for this case against the corresponding frequencies. As dotted lines are also plotted, the corresponding damping factors against frequency for the case of the same generator, the fundamental of which is critically damped by distributed resistance, $R=R_{n}=0.61 \mathrm{ohm}$.

It should be remarked that had we considered the constants of this generator to be lumped, we would have computed a terminal resistance

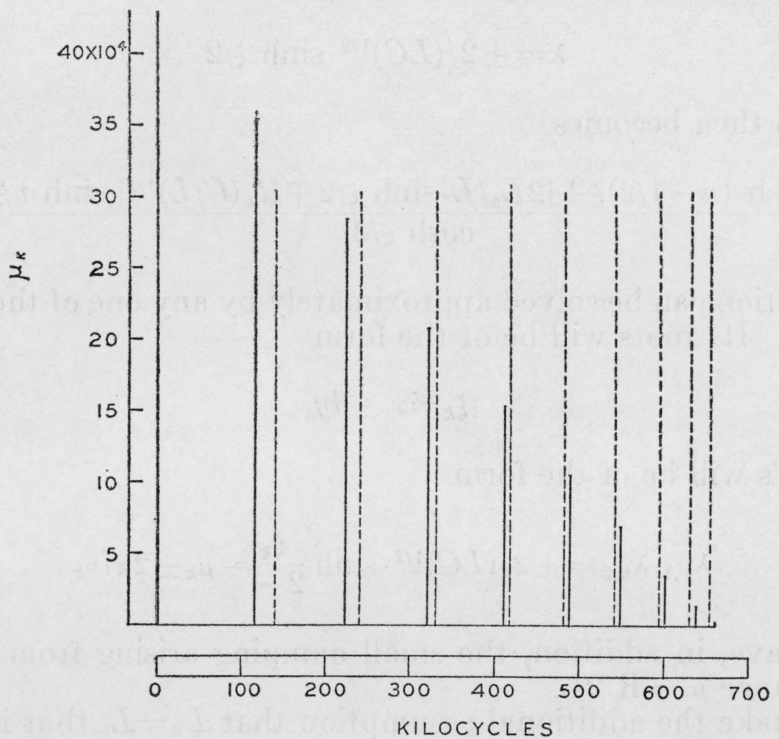

FigURE 6.-Damping factors against frequencies for a 10-section surge-current generator.

Solid lines, damping factors against frequencies for the case of a lumped terminal resistance just sufficient to critically damp the fundamental. $R=0.01 \mathrm{ohm}, R_{n}=2.3 \mathrm{ohms}$.

Dotted lines, damping factors against frequencies for the case of a distributed resistance just sufficient to critically damp the fundamental. $R=R_{n}=0.61 \mathrm{ohm}$.

of $4.1 \mathrm{ohms}$ necessary to critically damp the system. The damping resistance obtained from the present closer calculations, that is 2.3 ohms, is in reasonable agreement with the experimental values observed on just such a generator, that is 1.5 to $2.0 \mathrm{ohms}^{7}$

\section{CONCLUSION}

It is thus seen that the mathematical theory of surge-generators presents no essential difficulties, and that it is possible to set up complete formal solutions for both the surge-voltage and the surge-current generator which take account both of the internal structure of the generator and of the load to which it is connected. From these solutions it is possible to obtain exact numerical values for the damping factors and frequencies in certain special cases, and to obtain approximate values in all cases. Exact expressions for the amplitudes and phase angles can be obtained only in certain special cases.

\footnotetext{
7 P. L. Bellaschi, Elec. Eng. 53, 86 (1934).
} 
It has been the purpose of this discussion to outline the general method of attack on the mathematical problem presented by surgegenerator circuits and the difficulties which will be met rather than to discuss in detail all the pecial cases which might be derived from the equations given above. If a particular generator were under consideration, many valid assumptions and approximations leading to useful approximate solutions might suggest themselves, which it would be fruitless to consider in a general discussion.

Washington, July 23, 1936. 